\title{
Coding and Resource Scheduling in Packet Oriented Adaptive TDMA/OFDMA Systems
}

\author{
Tommy Svensson ${ }^{\dagger}$, Sorour Falahati* and Mikael Sternad ${ }^{*}$ \\ 'Dept. of Signals and Systems, Chalmers University of Technology, SE-41296 Göteborg, Sweden, \\ "Signals and Systems, Uppsala University, PO Box 528, SE-751 20 Uppsala, Sweden, \\ tommy.svensson@chalmers.se, \{sorour.falahati, mikael.sternad\}@signal.uu.se
}

\begin{abstract}
Within the EU FP6 Integrated Project WINNER, adaptive transmission is investigated as a key technology for boosting the spectral efficiency of a new radio interface for $4 G$ systems. Adaptive allocation and link adaptation of timefrequency chunks based on channel prediction in an OFDMbased system offers a significant potential to design a spectrally efficient system. The chunk size is typically defined based on the minimum coherence time and coherence bandwidth of the targeted channels. It is important to allow efficient channel coding and link retransmission schemes without restricting the resource scheduler, even for systems using small chunk sizes, to achieve multi-user diversity gains. In this paper we introduce some possible approaches to implement FEC coding and Hybrid ARQ and analyze their interplay with resource scheduling in packet oriented adaptive TDMA/OFDMA.
\end{abstract}

Index Terms-4G mobile wireless systems, adaptive transmission and multiple access, Orthogonal Frequency Division Multiplexing (OFDM), forward error correction coding (FEC), link retransmissions, spectral efficiency.

\section{INTRODUCTION}

A DAPTIVE systems allocate (schedule) time, frequency and antenna resources based on channel quality and user requirements. They enable efficient resource utilization and multi-user scheduling gains, when channels to different terminals fade independently. In systems based on time division multiple access/ adaptive OFDM (TDMA/OFDMA), time-frequency resources (chunks) are allocated to the individual users and link adaptation is performed individually in each chunk. The chunk size is chosen such that the channel is essentially flat in time and frequency. This provides a flexible small-scale granularity of the resources for multi-user scheduling and link adaptation, which makes it possible to obtain large multi-user diversity gains [1], [2], [3]. Based on the results obtained within the Swedish Wireless IP project ${ }^{1}$, we are currently evolving and assessing the feasibility of adaptive TDMA/OFDMA in novel broadband radio interfaces within the EU FP6 Integrated Project WINNER. We here investigate adaptive downlinks and uplinks based on fast scheduling and link adaptation, also for users at vehicular

\footnotetext{
${ }^{1}$ www.signal.uu.se/Research/PCCwirelessIP.html
}

speeds, with a non-adaptive diversity-based fall-back mode (diversity in time/frequency/space) for very fast moving users.

In the proposed downlink, each terminal predicts the signalto-interference-and-noise ratio (SINR) over a major part of the total bandwidth. All active terminals report source coded SINR values or source coded suggested modulation formats over a shared uplink control channel. A resource scheduler, located close to one or several radio access points, allocates the downlink resources.

In an adaptive uplink, the estimation and prediction of channels from each potential user could be problematic. In a system using frequency division duplex (FDD), the estimation must be carried out at the access point, and has to be based on pilots transmitted by all active terminals. To avoid unacceptable pilot overhead, these pilots must be transmitted simultaneously, by using overlapping pilots [4].

An open question raised here is how to best combine this fine-grained resource allocation and link adaptation with efficient channel coding schemes and retransmission protocols for network layer packets. An adequate combination preserves the multi-user scheduling and link adaptation gains regardless of various sizes of packets and different reliability requirements. This study proposes some approaches to tackle this problem, and initial results are presented. Related work can be found in e.g. [7] and [8].

Efficient FEC coding and Hybrid ARQ are important parts of an effective $4 \mathrm{G}$ air interface. FEC codes like Turbo codes and low-density parity-check (LDPC) codes typically need about thousand bits to perform efficiently. With small chunks, a strong outer FEC code has to span several chunks with dynamically varying capacity due to uneven SINR.

There are some possible approaches for combining coding and link adaptation:

- Adaptive coding and modulation within chunks

- Adaptive coding and modulation within chunks, combined with a strong concatenated outer FEC code

- Adaptive modulation (optionally combined with adaptive power fine tuning) within chunks, and the use of a strong concatenated outer FEC code.

In all cases, the rate limits for the link adaptation should be designed to take channel prediction errors into account [5]. In 
the first approach, used in [1], efficient per chunk link adaptation based on close to optimal rate limits, is possible with a few Modulation and Coding Schemes (MCSs). However, at low SINR or high prediction error variance, the code is forced to operate on a rather few channel bits with no diversity.

The two latter cases allow for a larger bit-interleaver spanning many chunks and an HARQ scheme to work together with the outer FEC code. Thus the ARQ mechanism can be decoupled from the chunk MCSs used in the previous transmission attempts. In the third approach mentioned above, employing simple soft bit demodulation of the chunks reduces the receivers complexity at the cost of larger granularity in the chunk link adaptation, unless a more fine-grained set of modulation alphabets is used.

If the link adaptation is good within the chunks, then an outer code will see a more or less AWGN like channel. Its design can thus be decoupled from the resource scheduling and link adaptation process, which simplifies the system.

We compare the above alternatives within a flexible architecture that allows small-grained resources, flexible multi-user scheduling and link adaptation, large FEC coding blocks and efficient (Hybrid) ARQ schemes to co-exist without restricting the potential gains of the individual techniques. We investigate these possibilities, quantifying pros and cons by measuring the throughput, bit error rate (BER), delay and by taking overhead such as control information and padding losses, into account.

\section{System Overview}

We explore adaptive TDMA/OFDMA designed at the carrier frequency $5 \mathrm{GHz}$. Adaptive transmission to vehicular users over TDMA/OFDMA downlinks has been earlier investigated in [2] and [3] for a more narrowband system of 5 MHz bandwidth at carrier frequency $1.9 \mathrm{GHz}$. Results in the WINNER context with a different design are given in [1], where more details on the channel predictors are presented.

The basic time-frequency resource unit, chunk, consists of a rectangular time-frequency area that comprises a number of subsequent OFDM symbols and a number of adjacent subcarriers, and is allocated exclusively to one user data flow. In Table I, we show the assumed chunk size along with important system parameters.

A chunk contains payload symbols and pilot symbols. The feedback loops for the FDD system is designed to be as fast as possible, under realistic constraints imposed by computation times and signaling delays, see [6] for more details. However, channel prediction is needed for scheduling and link adaptation, since extrapolating the present channel estimate would lead to large performance losses for vehicular users. Thus, in addition to channel estimation in coherent reception, the pilots are used for channel prediction for resource allocation and link adaptation. The chunks may also contain control symbols to minimize feedback delays, i.e. in-chunk control signaling.
TABLE I

BASIC PARAMETERS FOR FDD WIDE-AREA DOWNLINK AND UPLINK

\begin{tabular}{|c|c|}
\hline Centre frequency & $5.0+/-0.384 \mathrm{GHz}$ \\
\hline Number of OFDM sub-carriers & 1024 \\
\hline FFT BW & $20.0 \mathrm{MHz}$ \\
\hline Signal BW & $16.25 \mathrm{MHz}$ paired \\
\hline Number of used subcarriers & $19531 \mathrm{~Hz}$ \\
\hline Sub-carrier spacing & $51.20 \mu \mathrm{s}$ \\
\hline OFDM symbol length (excl. CP) & $5.00 \mu \mathrm{s}$ \\
\hline Cyclic prefix (CP) length & $156.24 \mathrm{kHz} \times 337.2 \mu \mathrm{s}$ \\
\hline Physical chunk size & $8 \times 6=48$ \\
\hline Chunk size in symbols &
\end{tabular}

The number of offered payload bits in a chunk depends on the utilized MCS, which is selected adaptively. If the same MCS set is used as in [1], ranging from BPSK rate $1 / 2$ to $64-$ QAM rate $5 / 6$, the number of payload bits per chunk varies between 18 and 180. Thus, a large FEC block typically has to span several chunks.
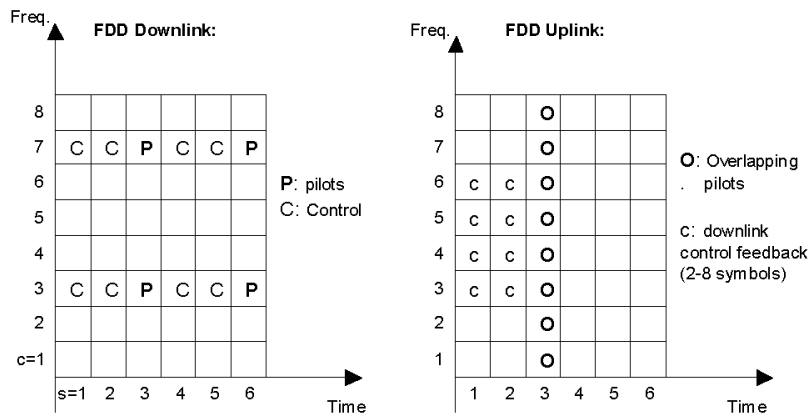

Fig. 1. Pilot and control symbol patterns in FDD downlink and uplink.

\section{A. Downlink}

The downlink is designed as follows. Regular pilot patterns are transmitted on the downlink. Based on channel measurements up to chunk time $i$, all active terminals predict the channel quality in all chunks within a sub-band of interest at the future chunk time $i+2$. These reports are source-coded and transmitted on uplink control symbols within the uplink chunks at time $i+1$. The appropriate MCS that could be used by each terminal in each chunk is then determined based on SINR rate limits. The adaptive resource scheduler at the base station allocates each chunk at time $i+2$ exclusively to one of the flows. The allocation is reported by control symbols placed within the allocated downlink chunks at time $i+2$.

The control symbols are all located on the same sub-carriers as the pilots (see Fig. 1 (left)), to simplify use of them for decision-directed channel estimation.

\section{B. Uplink}

On the uplink, terminals taking part in adaptive transmission are in competition for a part of the total 16.25 $\mathrm{MHz}$ band, called a competition band. All active terminals assigned to a competition band simultaneously send overlapping pilot signals during chunk time $i$. All eight symbols within OFDM symbol $s=3$ as shown in Fig. 1 (right) are reserved for this purpose. Predictors located at the access point predict the channels for all terminals at time $i+2$. The prediction is based on the latest and previously received signals at the locations of the overlapping pilots. The 
appropriate MCS that could be used by each terminal in each chunk is then determined. The adaptive resource scheduler assigns the uplink transmission for time $i+2$ and informs the terminals by in-chunk signaling using control symbols of the downlink chunk at time $i+1$.

The in-chunk control symbols on the uplink that are positioned early in the chunk, (see Fig. 1 (right)), are part of the control loop for the downlink. They carry the downlink channel prediction reports from all active terminals. The use of overlapping pilots in a Kalman filter that simultaneously estimates and predicts all channels is described in [4].

\section{MAC ARCHITECTURE}

The media access control (MAC) architecture in Fig. 2 is a framework capturing all the possible coding and link adaptation approaches outlined in the introduction. When the Radio Link Control (RLC) Protocol Data Units (PDUs) are entering the MAC, they could be segmented, depending on their size, into ReTransmission Units (RTUs) appropriate for the ARQ. After attachment of CRC, these RTUs are then optionally $\mathrm{FEC}$ encoded with an outer code, bit-interleaved and punctured to produce incremental redundancy for the later use by a Hybrid ARQ scheme.

The optionally encoded segments, are queued per flow in the resource scheduling buffer. These queues are then drained with bit-level granularity. For each buffer, there is one resource scheduler that determines which queues are to be drained, and to what extent. The resource scheduler works on the time-scale of the chunk duration. The resource scheduler optimizes the allocation of physical channel resources under certain constraints such as interference avoidance. Since adaptive transmission is feasible up to a limiting velocity (around $70 \mathrm{~km} / \mathrm{h}$ ) or down to a certain average user SINR [1], the resource scheduler performs adaptive scheduling when feasible. Otherwise non-frequency-adaptive scheduling uses averaging with respect to frequency-selective channel variations by mapping onto sub-carriers in well dispersed chunks.

The scheduled bits are mapped either directly by adaptive modulation or adaptive coding and modulation with bitinterleaving onto the physical channel resource units, i.e chunks (in general chunk layers when more than one spatial dimension is utilized in multi-antenna transmitters).

\section{Simulation Results for FDD Downlink WIth DIFFERENT FEC CODING APPROACHES}

In this section, we compare two of the different approaches for FEC coding and link adaptation as outlined in the introduction. The throughput, multi-user scheduling gain and bit error rate (BER) are measured for different terminal speeds taking prediction uncertainty into account.

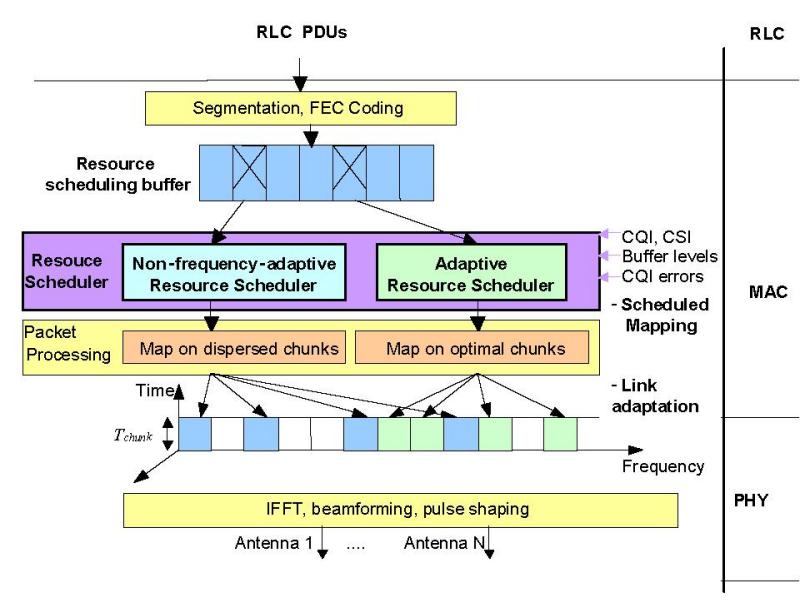

Fig. 2. MAC Architecture.

TABLE II

WINNER URBAN MACRO POWER DELAY PROFILE

$\tau_{n}=\left\{0,10,30,250,260, \quad p_{n}=-\{3,5.2,6.98,4.72,6.94,8.70,5.22\right.$, $280,360,370,385,1040, \quad 7.44,9.20,8.19,10.41,12.17,12.05,14.27$, $1045,1065,2730,2740$, $2760,4600,4610,4625\} \mathrm{ns}$ $16.03,15.50,17.72,19.48\} \mathrm{dB}$

In the multilink simulations, all the Rayleigh fading channels are modeled by the WINNER Urban Macro Power Delay profile, see Table II, and have the same statistical properties. The terminals are full duplex and all have the same velocity. The interference is modeled by white Gaussian noise and all terminals have the same average SINR, to facilitate interpretation of the results.

One competition band spanning the whole system bandwidth is assumed. The scheduling strategy used is Proportional Fair, which in this case, where all users have the same average SINR, reduces to the Max Throughput strategy of giving the chunk to the user who can use the highest modulation-coding rate. The resource scheduling buffers are never emptied.

The channels are not perfectly flat within the chunks: there is in general variability both in the time direction and in the frequency direction. Within each chunk, the modulation and coding scheme potentially used by each user is determined by taking the average predicted SINR, $S I N R_{a v}$, and the predicted SINR at the worst point within the chunk, $S I N R_{w}$, for that user. The weighted average is used as the effective SINR: $\operatorname{SINR}[d B]=b \operatorname{SINR}_{a v}[d B]+(1-b) \operatorname{SINR}_{w}[d B]$. The effect of channel estimation errors on the demodulation is not considered. With an outer code, it is expected that it is possible to put a large weight on the average SINR, i.e. $b$ close to 1 , because of the bit-interleaver spanning many chunks.

We use 512-bit and 1024-bit packets. The overhead due to $\mathrm{CRC}$ code and sequence numbers for $\mathrm{ARQ}$ is not taken into account. Each packet is distributed among the allocated chunks, and superfluous payload symbols are filled with zeros. Here we show results for two cases:

1. "Inner code" case: Adaptive coding and modulation in each chunk, with the following 
MCS set: BPSK rate $1 / 2$, QPSK rate $1 / 2$, QPSK rate 3/4, 16-QAM rate $1 / 2,16$-QAM rate $2 / 3,16-$ QAM rate 5/6, 64-QAM rate 2/3 and 64-QAM rate $5 / 6$, based on the rate $1 / 2$ convolutional code with constraint length 9 , and generator polynomials $(561,753)$ in octal representation. This case is the same as investigated in [1].

2. "Outer code" case: Adaptive modulation in each chunk taken from BPSK, QPSK, 16-QAM and 64QAM and an outer FEC code with adaptive puncturing. The outer code is chosen to be the same rate $1 / 2$ convolutional code as above and is adaptively punctured in each chunk using the same puncturing matrices as in the Inner code case.

Note that both cases use the same modulation constellations, the same puncturing in each chunk and the same convolutional code. Bit-interleaving within chunks is also made in both cases before mapping onto the modulation symbols. The difference is the larger FEC block and the extra bit-interleaver over the chunks in the "Outer code" case.

If all bits belonging to a packet are received correctly, then the packet is released to higher layers, otherwise a link retransmission would occur. However, link level retransmissions are not used in the given simulation results. The throughput is defined as the number of payload bits in the received packets divided by the total number of transmitted payload symbols.

The rate limits are optimized under a maximal bit error rate constraint of 0.001 , for a given average SINR and prediction error variance [5], and the same rate limits are used for both the Inner code case and the Outer code case. The prediction normalized MSE (NMSE) values are taken from [1]. The actual average bit error rate becomes lower, since the maximal BER is targeted at the lower rate thresholds.

The dashed curves in Fig. 3-5 show the performance in the "Inner code" case and the solid curves show the performance in the "Outer code" case. The rate limits are designed to attain the BER constraints in the presence of prediction errors, and the effective SINR is based on the effective SNR expression above, with $b=0.4$ or $b=1$. Note that the prediction uncertainty decreases with the number of active users since with many users, chunks are given to users with relatively good channels having a small prediction uncertainty.

The "Outer code" case is shown to give a gain in this comparison. As seen in Fig 3, when using the same packet size and $b$ parameter value, there is an increase in throughput with the Outer code. This is due to the lower tail bits overhead in the Outer code case. The resulting BER and PER becomes smaller for the Outer code case, and that can be used to set a more aggressive value for parameter $b$, and still fulfill the target BER. As a result, a higher effective SINR will be used in the link adaptation, and there is the potential for larger throughput. We tested $b=1$, and as seen in Fig. 4, with that value we still satisfy the target BER. In Fig. 3, a substantial increase in throughput is seen for this $b$-value with the Outer code, especially at low SINR. (The maximum throughput of the MCSs is a limiting factor at high SINR.)

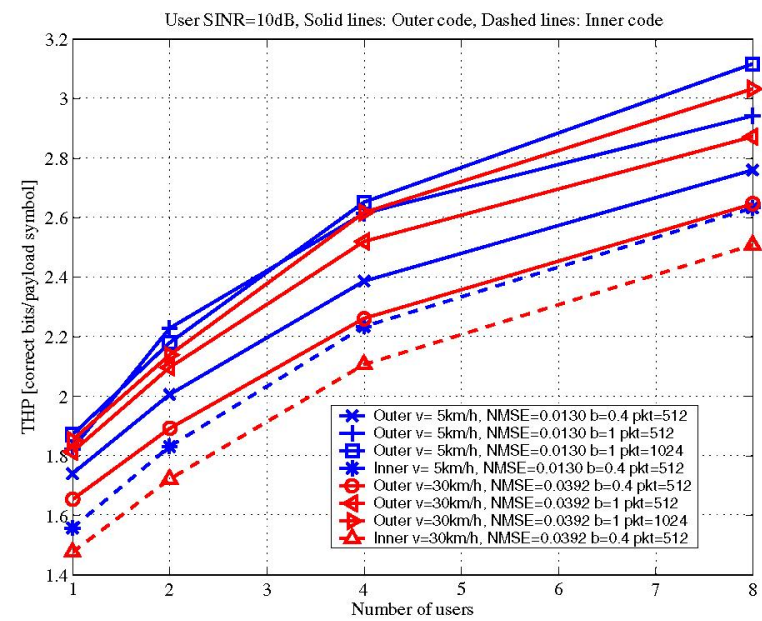

User SINR=19dB, Solid lines: Outer code, Dashed lines: Inner code

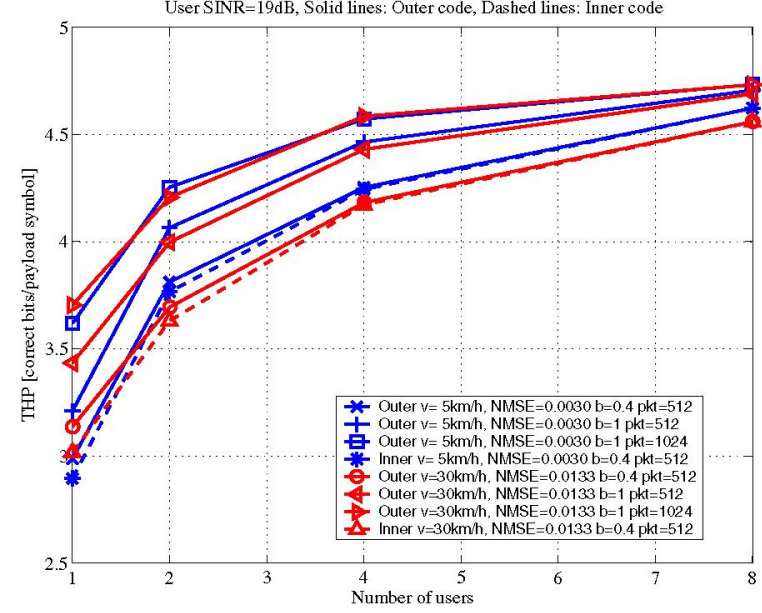

Fig. 3. Throughput as a function of the number of active users, all with the same average SINR of $10 \mathrm{~dB}$ (top) and $19 \mathrm{~dB}$ (bottom) in FDD wide-area downlink at user velocities $5 \mathrm{~km} / \mathrm{h}$ and $30 \mathrm{~km} / \mathrm{h}$. Prediction uncertainty with the given NMSE is taken into account and chunk SINR is calculated with $b=0.4$ and $b=1$ respectively for two different packet sizes. Dashed lines represent the "Inner code" case and solid lines represent the Outer code.

In Fig. 3-5, we also show the performance with $b=1$ and a larger packet size of 1024 bits with the Outer code. Only with low SINR and one user, the bit error rate is little larger than the target for this packet size, and there is a substantial increase in throughput even with this simple convolutional code as the outer code. In Fig. 4, we show the resulting packet error rate (PER) for the investigated cases. The suitable PER values depends on the design of the (Hybrid) ARQ scheme, which is for further study.

\section{Conclusions}

In this paper, we outlined a flexible MAC layer and discussed different possibilities to perform efficient FEC coding and at the same time maintain large multi-user diversity gains for adaptive TDMA/OFDMA systems using channel prediction. We investigated two cases, chunk based adaptive coding and modulation and an outer code followed by adaptive puncturing and adaptive modulation in each chunk. We found that there is a substantial gain already with a simple outer code. 

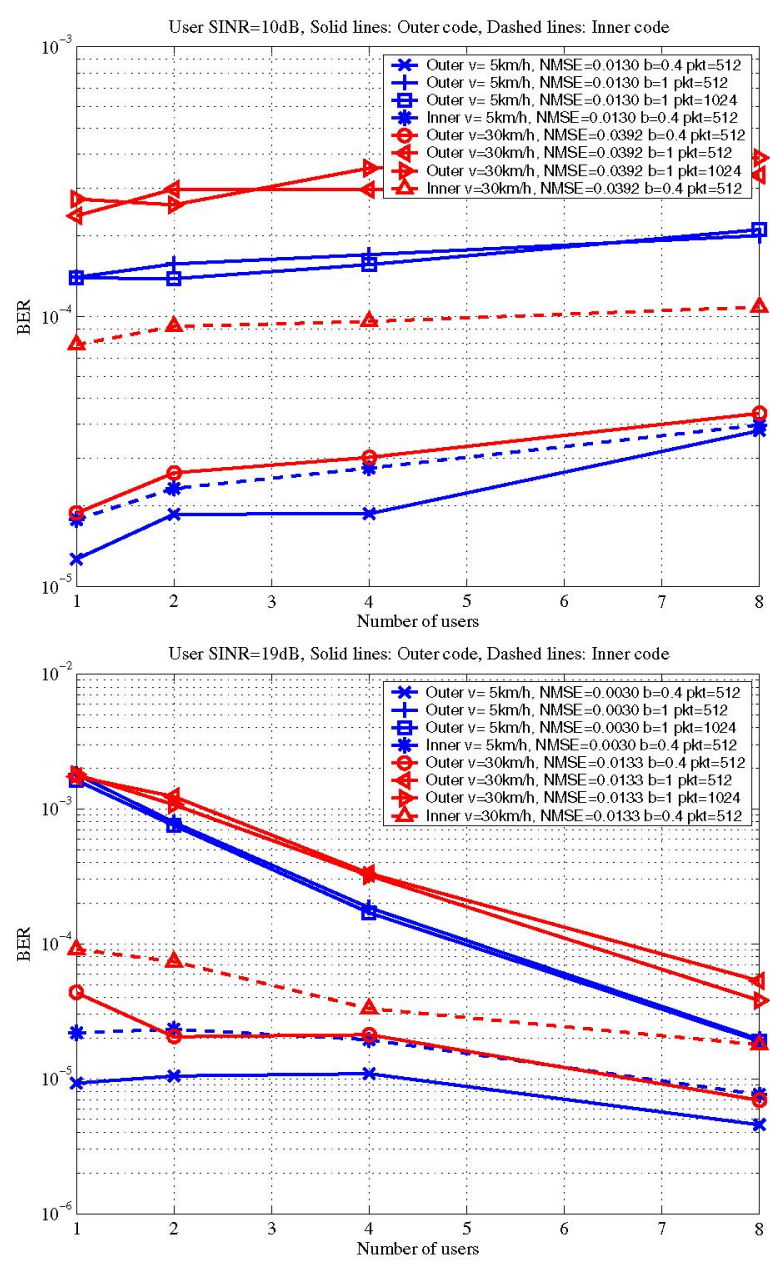

Fig. 4. Bit error rate as a function of the number of active users, all with the same average SINR of $10 \mathrm{~dB}$ (top) and $19 \mathrm{~dB}$ (bottom), and with the same scenario parameters as in Fig 3 .

\section{FUture WORK}

The presented results are part of an ongoing extensive study within the WINNER II project. We quantify the gain of using a stronger outer $\mathrm{FEC}$ code, using a more fine-grained set of modulation constellations and power-tuning to target the ratelimits. We compare these more advanced outer code schemes with the results presented here, both with respect to throughput and their expected performance with (H)ARQ. Furthermore, we elaborate more on the performance with different packet sizes and appropriate rate limits in the adaptive schemes.

\section{ACKNOWLEDGMENT}

This work was performed in the framework of the IST projects IST-2003-507581 WINNER and IST-4-027756 WINNER II, which are partly funded by the European Union. The authors would like to acknowledge the contributions of their colleagues.
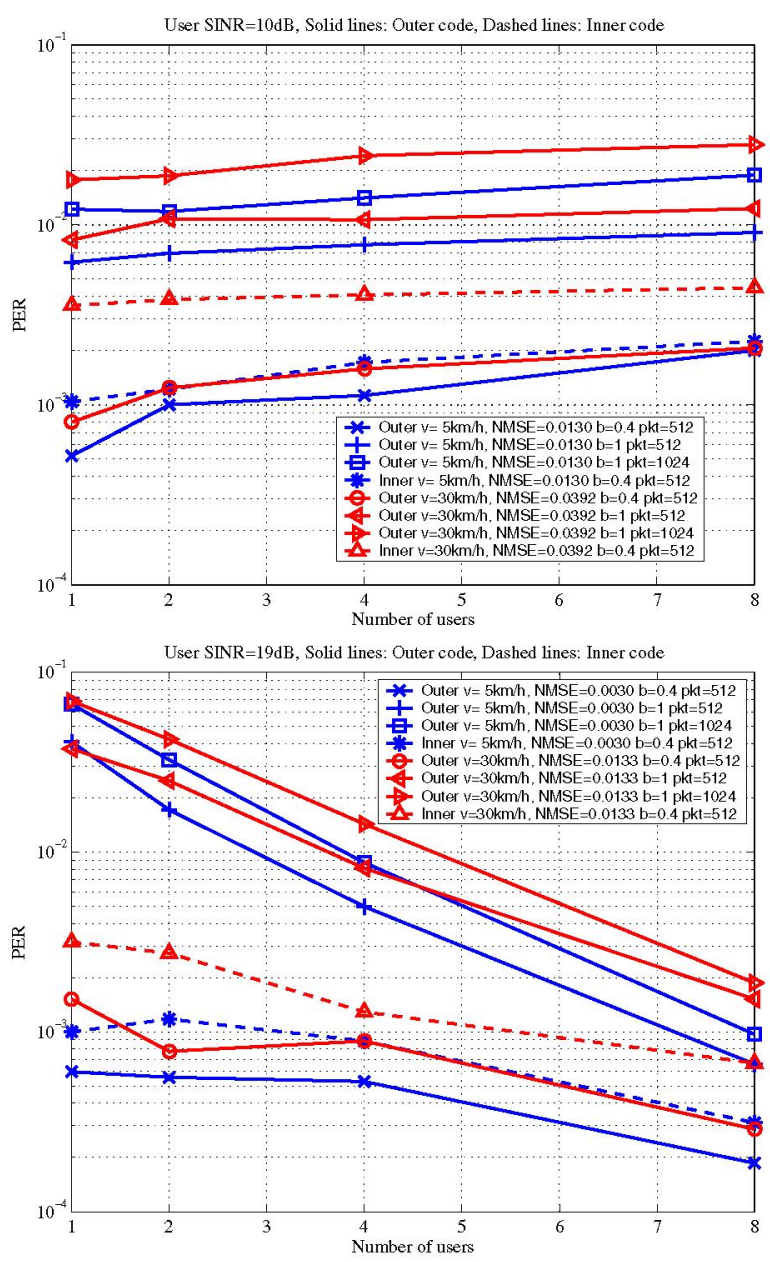

Fig. 5. Packet error rate as a function of the number of active users, all with the same average SINR of $10 \mathrm{~dB}$ (top) and $19 \mathrm{~dB}$ (bottom), and with the same scenario parameters as in Fig 3.

\section{REFERENCES}

[1] M. Sternad, S. Falahati, T. Svensson, D. Aronsson. "Adaptive TDMA/OFDMA for Wide-Area Coverage and Vehicular Velocities," 14th IST Mobile \& Wireless Communications Summit, Dresden, Germany, June 2005.

[2] W. Wang, T. Ottosson, M.Sternad, A. Ahlén and A. Svensson, "Impact of multiuser diversity and channel variability on adaptive OFDM," VTC 2003 Fall, Orlando, FL, Oct. 2003.

[3] M. Sternad, T. Ottosson, A. Ahlén and A. Svensson, "Attaining both coverage and high spectral efficiency with adaptive OFDMA downlinks," VTC 2003-Fall, Orlando, Fla, Oct. 2003.

[4] M. Sternad and D. Aronsson, "Channel estimation and prediction for adaptive OFDMA/TDMA uplinks, based on overlapping pilots", International Conference on Acoustics, Speech and Signal Processing (ICASSP 2005). Philadelphia, PA, USA, March 19-23 2005. Online: http://www.signal.uu.se/Publications/abstracts/c0501.htm1

[5] S. Falahati, A. Svensson, T. Ekman and M. Sternad, "Adaptive modulation systems for predicted wireless channels," IEEE Trans. on Communications, vol. 52, Feb. 2004, pp. 307-316.

[6] IST-2003-507581 WINNER, "D2.4 Assessment of adaptive transmission technologies", February, 2005.

[7] B. Classon, K. Blankenship and V. Desai, "Channel coding for 4G systems with adaptive modulation and coding," IEEE Wireless Communications, April 2002.

[8] A. Talukdar, P. Sartori, M. Cudak, B. Classon and Y. Blankenship, "Aggressive modulation/coding scheme selection for maximizing system throughput in a multi-carrier system," IEEE VTC 2005-Spring, Stockholm. 